\title{
Article
}

\section{Phase Change and Regeneration Behaviors of Amino Acid Ionic Liquids Absorbents}

\author{
Xueqin Chen 1, Guo Hui ', Huang Ouyang ', Qiongfang Song 1, Zhengguang Sun ', \\ Ziqiang $\mathrm{Xu}{ }^{1}$, Cao Li ${ }^{1,2 *}$, Bingbing Jiang ${ }^{2,3^{*}}$ \\ 1 Key Laboratory of Polymer Material in Hubei Province, Hubei University, Wuhan 430062, China \\ 2 Hubei Collaborative Innovation Center for Advanced Organic Chemical Materials, Wuhan 430062, China \\ 3 Key Laboratory of Green Preparation and Application for Functional Materials, Ministry of Education, \\ Hubei University, Wuhan 430062, China
}

*Correspondence: bingbingjiang@hubu.edu.cn; Tel: 86-027-88661637, Fax: 86-027-88661637

\# Cao Li, Email: licao0415@163.com; Tel: 86-027-88661729

\begin{abstract}
As novel materials for carbon capture, phase change solvents can separate into two immiscible phases during the $\mathrm{CO} 2$ capturing procedure under a certain temperature. The solvent systems can significantly decrease the energy consumption since the solvents can be regenerated by only heating the rich-CO2 phase. In this work, amino acid ionic liquids (AAILs) were synthesized using quaternary ammonium salts and amino acids as raw materials, and the aqueous solutions were prepared as novel liquid-solid phase change solvents. The results showed that the solvents had excellent $\mathrm{CO} 2$ absorption capacity, and the AAILs functionalized by glycine and tryptophan exhibited significant phase change properties. The mechanism of phase-change of the solvent were mainly due to the lower solubility of the product after reaction between AAILs and CO2. The solvent with tryptophan as anion could be regenerated by only heating the $\mathrm{CO} 2$-riched solid phase, which might significantly decrease energy consumption of regeneration. And the absorbent could be reused with the regenerated absorption ratio up to $79 \%$. The solvent system has great potential in industrial application due to the easy operation process and efficient recycling ability.
\end{abstract}

Keywords: Amino acid ionic liquid; phase change solvent; high $\mathrm{CO} 2$ absorption capacity; mechanism of phase change; recycling ability

\section{Introduction}

Large emission of $\mathrm{CO} 2$ from the industrial combustion of fossil fuels has caused increasing $\mathrm{CO} 2$ concentration in the atmosphere and the greenhouse effect[1]. Therefore, carbon capture and sequestration(CCS) technology is highly valued and a mass of researchers devote to find the materials that can meet the demand of $\mathrm{CO} 2$ capture with efficient gas absorption, low energy consumption, eco-friendly property and industrial adaption.

Aqueous solutions of alkanolamine or their mixtures are one of the effective methods to capture $\mathrm{CO} 2$ in industry. However, the amine system presents some drawbacks, such as corrosion of the equipment, prone to degrade under high temperature and oxidative degradation and high volatility[2]. Amino acid salt (AAS) is one of the alternative solvents for $\mathrm{CO} 2$ capture, since they are easily obtained, biocompatible and biodegradable and desirably resistant to degradation[3]. Recently, some amino acid salt solvents have been applied for $\mathrm{CO} 2$ separation from mixture gas[4,5]. However, one of the potential shortcomings of amino acid salt is that $\mathrm{CO} 2$ absorption capacity of the solvent would decrease after multiple regeneration cycles[6]. Ionic liquids (ILs) considered as "green" solvents have attracted more and more attention for their outstanding physicochemical properties, such as negligible vapor pressures, high thermal stabilities, and tunable properties[7-13]. Of all the 
features, the most attractive one of ILs is the ability to modify both the cationic and anionic components contributing to a specific application. ILs not only possesses significant $\mathrm{CO} 2$ physical solubility but also exhibit strong chemical affinity to $\mathrm{CO} 2$ by the functionalization of cations or anions[14-17]. Therefore, amino acid ionic liquids (AAILs) are one type of the functional ionic liquids that could capture and separate $\mathrm{CO} 2$ from biogases. They possess the excellent properties of ILs, and can be obtained by a relatively simple synthesis via the neutralization reaction of a cationic hydroxide solution and amino acid, which make the preparation of AAILs inexpensive and eco-friendly[18-20]. Most AAILs in neat form are either solids or liquids with extremely high viscosity up to $4180 \mathrm{mPa} s$ at room temperature[21]. Adding AAILs into the MDEA aqueous solution can increase the absorption rate of $\mathrm{CO} 2$ dramatically and enhance transfer process in MDEA solution[22]. Blending AAILs with water and/or other organic solvents to form mixed absorbents would downgrade the viscosity and the cost[23]. However, the high viscosity limits the usage of AAILs as CO2-capture solvents.

A new breed of $\mathrm{CO} 2$ absorption systems-phase change solvents has drawn much attention recently[24-26]. Upon CO2 loading, the mixed system of a tertiary amine and diamine formed two immiscible liquid phases-the heavy $\mathrm{CO} 2$ rich phase and the $\mathrm{CO} 2$ lean phase. The $\mathrm{CO} 2$ rich phase was picked up and heated to desorption temperature, illustrating the potential for reduction in regeneration heat through desirable cyclic capacity and very high CO2 stripping pressures[27].

Normally, precipitation is avoided in the $\mathrm{CO} 2$ capture process. However, if the precipitant contains $\mathrm{CO} 2$ (i.e., carbamate, carbonate/bicarbonate) and most of the $\mathrm{CO} 2$-capturing chemicals, lower energy consumption for the solvent regeneration would be achieved by only desorping the precipitate phase for solvent recovery [29]. Triethylenetetramine/ethanol system[30] produced a solid precipitate after capturing $\mathrm{CO} 2$, which can be easily separated and regenerated. $81.8 \%$ of the total $\mathrm{CO} 2$ capture amount was in the solid phase in the formation of TETA-carbamate, which can be completely de-composited at $90 \mathrm{oC}$. Amino acid salt solvents turned into a CO2-lean phase and a CO2-rich phase, which contained $90 \% \mathrm{CO} 2$ of the total capture amount. The dominant component of the $\mathrm{CO} 2$-rich phase was bicarbonate[31]. Since the alkali carbonate/bicarbonate couldn't wholly decomposite and regenerate at the desorption temperature, the AAs / $\mathrm{H} 2 \mathrm{O}$ system didn't possess the multiple regeneration ability.

In this work, we synthesized different kinds of hydrophilic amino acid liquids (AAILs) that were functionalized based on quaternary ammonium cations with anions of glycine(Gly), L-histidine (LHis), L-aspartic acid (DL-Asp) and L-tryptophan (L-Trp) respectively. Then, the CO2 absorption experiment was carried out with the aqueous solutions made from AAILs prepared. Phase change was found in the AAILs/H2O absorbent during the $\mathrm{CO} 2$ bubbling process, particularly, there appeared a quick precipitation in saturated aqueous solution of [TMA][Trp] with a large amount of powder precipitated. The favorable regeneration properties of the solid phase illustrated that [TMA][Trp] was a novel reversible absorbent with low energy consumption of regeneration. 


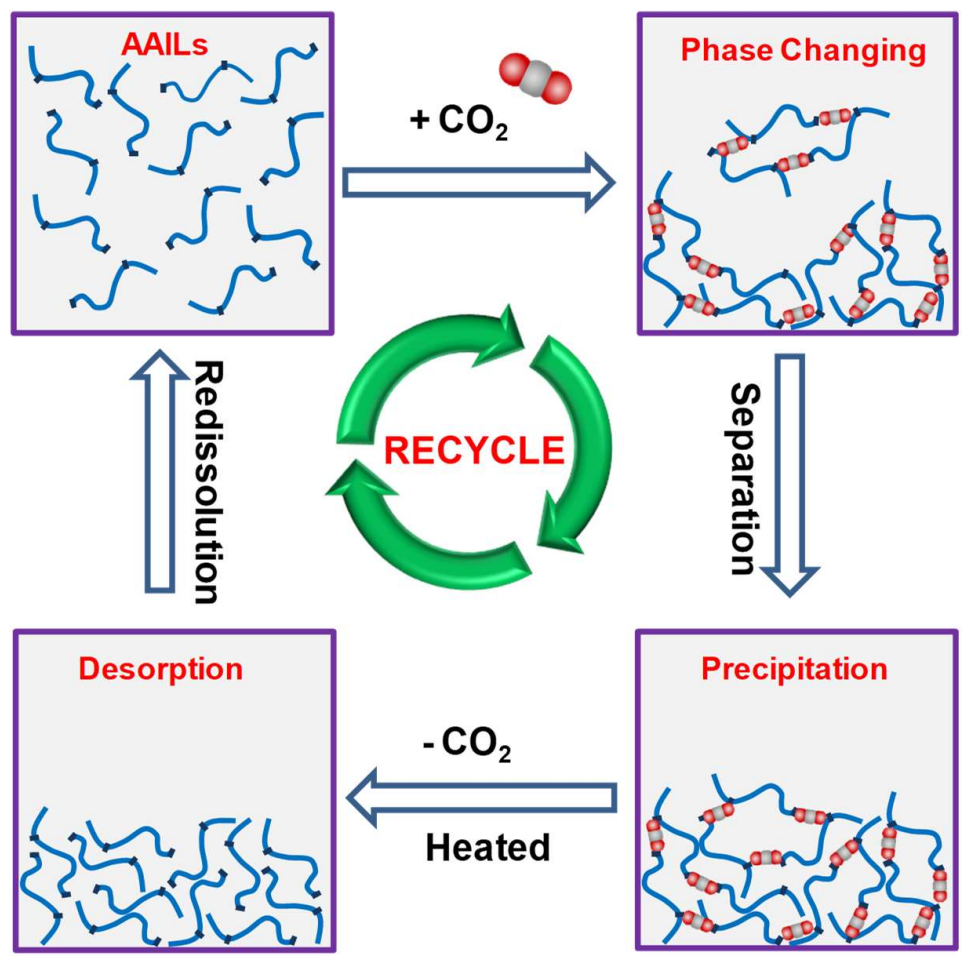

Scheme 1. CO2 absorption, desorpiton and reuse of AAIL-based phase-changing solvent.

\section{Experimental PART}

\subsection{Materials}

Analytical grade choline chloride (purity=99\%), tetramethylammonium chloride(purity=99\%), benzyltrimethylammoniumchloride (purity=99\%), trimethyl-octylammonium chloride(purity=99\%) were supplied by Sinopharm Chemical Reagent Co., Ltd. Analytical grade glycine(purity>99\%), Lhistidine (purity=99\%), L-aspartic acid(purity=98\%), L-tryptophan (purity=99\%) were supplied by Hangzhou Lanbo Industrial Co., Ltd. CO2 (purity $>99.9 \%$ ) was purchased from Wuhan Xiangyun industry \&trade Co., Ltd.

\subsection{Synthesis of amino acid ionic liquids (AAILs)}

The amino acid ionic liquids were synthesized via a two-step process: ion exchange, neutralization, as illustrated in Fig 1.

A mixture of tetramethylammonium chloride* (TMAC, $0.1 \mathrm{~mol}$ ) and potassium hydroxide $(\mathrm{KOH}, 0.1 \mathrm{~mol})$ in absolute ethyl alcohol $(\mathrm{EtOH})$ was reacted at room temperature for 3 hours in a flask equipped with a magnetic stirrer. After filtering to remove $\mathrm{KCl}$ under N2 atmosphere, the tetramethylammonium hydroxides ([TMA][OH])-Ethanol solution was concentrated through rotation evaporation at $35 \mathrm{oC}$.

[TMA][AA] ILs were produced by adding a slight excess of amino acid into the concentrated [TMA] [OH] solution, stirring at 40 oC under N2 atmosphere for 24 hours. After rotation evaporation at $50 \mathrm{oC}$, the residual solution containing the ionic liquids was dried in vacuum at $60 \mathrm{oC}$ for 48 hours, and the crude products were obtained. In order to get final pure amino acid ionic liquids, ethanol was added into the crude products to precipitate the excess amino acids. After filtration, the solution was rotary evaporated to remove ethanol. Finally, the product was obtained by drying at $60 \mathrm{oC}$ under vacuum for at least 48 hours[32].

* Take [TMA][AA] as an example of the synthesis of amino acid ionic liquids. Other amino acid ionic liquids used in this work were produced in the same way. 


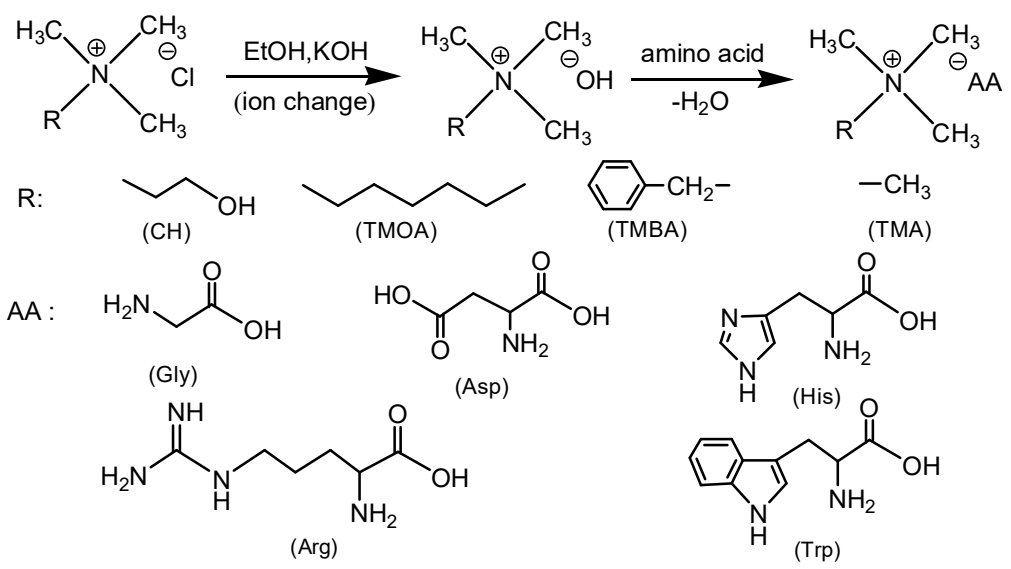

Figure 2. Schematic Diagram of Synthesis of the Amino Acid Ionic Liquids (AAILs).

\subsection{Characterization}

The chemical structures of synthesized AAILs were characterized by 1H-NMR (Varian Inova, $600 \mathrm{MHz}, \mathrm{D} 2 \mathrm{O}$ ) and FT-IR (Prestige-21). 1H-NMR was tested in room temperature and the solvent was D2O, with four methyl silane as internal standard. For the FT-IR measurement of AAILs, an ethanol solution and a disk made of $\mathrm{KBr}$ were used. $0.5 \mathrm{~g} \mathrm{KBr}$ and $5 \mathrm{mg}$ dry sample were mixed in a mortar and then, fully ground pressure at 1.5 bars for 1 minute. The test was conducted in room temperature with scanning range of $400 \sim 4000 \mathrm{~cm}-1$.

Thermogravimetric analysis (TGA) of AAILs was performed on a METTLER TOLEDO. The sample was heating from $30 \mathrm{oC}$ to $600 \mathrm{oC}$ with the heating rate of $5 \mathrm{~K} /$ min under Nitrogen atmosphere, of which the purity was $99.999 \%$. And the Nitrogen velocity was $50 \mathrm{~mL} / \mathrm{min}$.

\section{4. $\mathrm{CO} 2$ Absorption and Desorption Experiment}

\subsection{1. $\mathrm{CO} 2$ absorption and phase change}

$\mathrm{CO} 2$ absorption experiment was performed by bubbling experiment and $\mathrm{CO} 2$ absorption capacity was defined as the amount of $\mathrm{CO} 2$ per mole AAILs absorbed. How many $\mathrm{CO} 2$ the absorbent ca ptured can be obtained by measuring the mass change of the device after and before the $\mathrm{CO} 2$ bubbling $(\Delta \mathrm{m})$. The schematic diagram of bubbling device was shown in Fig 3.

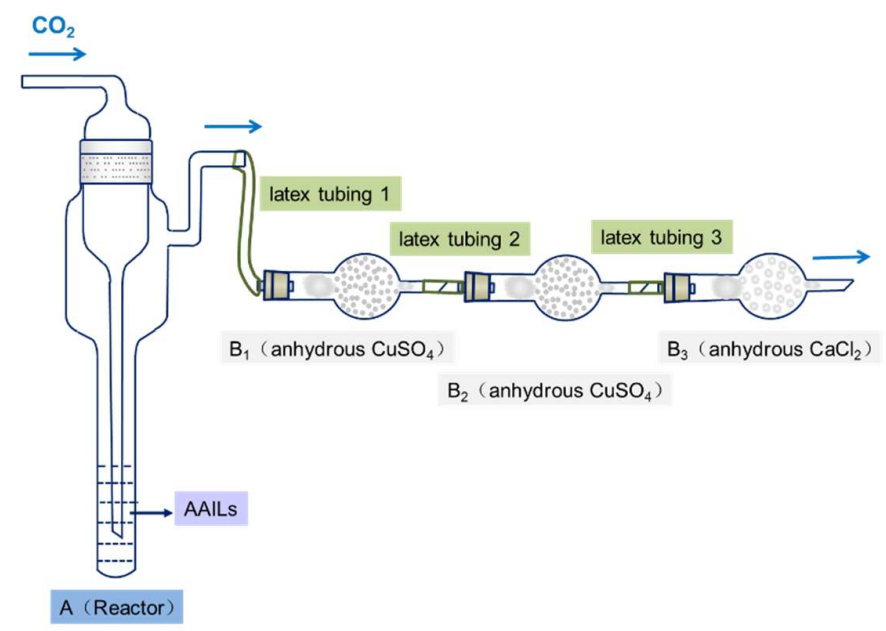

Figure 3. Schematic diagram of $\mathrm{CO} 2$ bubbling device.

Absorbent was added into the Reactor (container, A), in which CO2 reacted with the solvent. Drying tubes- $\mathrm{B} 1$ and $\mathrm{B} 2$ were both filled with anhydrous $\mathrm{CuSO} 4$ in order to catch $\mathrm{H} 2 \mathrm{O}$, while drying 
tube $\mathrm{B} 3$ was filled with anhydrous $\mathrm{CaCl} 2$. $\mathrm{CO} 2$ absorption capacity can be calculated according to the following formula:

$$
A=\frac{n_{C O 2}}{n_{\text {AAILS }}} \times 100 \%=\frac{M_{\text {AALLS }} \times\left(m_{a}-m_{b}\right)}{44 \times m_{\text {AAILS }}}=\frac{1000 \times\left(m_{a}-m_{b}\right)}{44 \times V \times C_{\text {AAILS }}}
$$

where MAAILs is the molecular weight of AAILs, mAAILs is the weight of AAILs in the solvent, CAAILs and $\mathrm{V}$ are the concentration and volume of the absorbent respectively, ma and $\mathrm{mb}$ are the weight of A, B1, B2 and latex 1-3 after and before bubbling respectively.

Here, the phase change efficiency is defined as the ratio of solid phase volume to the volume of whole absorbent. Then, the phase change efficiency can be estimated by the equation.

$$
\mathrm{w}=\frac{V_{s}}{V} \times 100 \%
$$

where $\mathrm{w}$ is the phase change efficiency, Vs is the volume of solid phase when the phase separation has done, and $\mathrm{V}$ is the volume of the whole absorbent.

\subsubsection{Regeneration of AAIL solvents}

[TMA][AA] was dissolved in deionized water to prepare a saturated aqueous solution at room temperature for the $\mathrm{CO} 2$ absorption experiment. The $2.5 \mathrm{M}$ aqueous solutions of $[\mathrm{CH}][\mathrm{Gly}]$, [TMA][Gly], [TMBA][Gly], and [TMOA][Gly] were also prepared for CO2 uptake, respectively.

The candidate solutions were loaded in a CO2 bubbling reactor as shown in Fig 3. All of the CO2 uptake experiments were carried out in room temperature. During the absorption/desorption cycling process, the phase change solvent with $\mathrm{CO} 2$ was centrifuged to accelerate the separation of the two phase. The precipitations carried $\mathrm{CO} 2$ were heated to the desorption temperature to release the uptake $\mathrm{CO} 2$, while the clear phase was pipetted out and added back to the $\mathrm{CO} 2$ bubbling reactor without any operation for next $\mathrm{CO} 2$ absorption experiment. Pure $\mathrm{CO} 2$ gas was used in all the absorption experiments. The $\mathrm{CO} 2$ absorption capacity of the solvent was measured by analytical balance. In this work, two methods were conducted for stripping $\mathrm{CO} 2$. One method is by heating the whole absorbent captured $\mathrm{CO} 2$ in $100 \mathrm{oC}$ for 6 hours, and another is by only heating the solid phase in 120 oC for 6 hours.

\section{Results and discussion}

\subsection{Characterization of AAILs (take [TMA][Gly] as an example)}

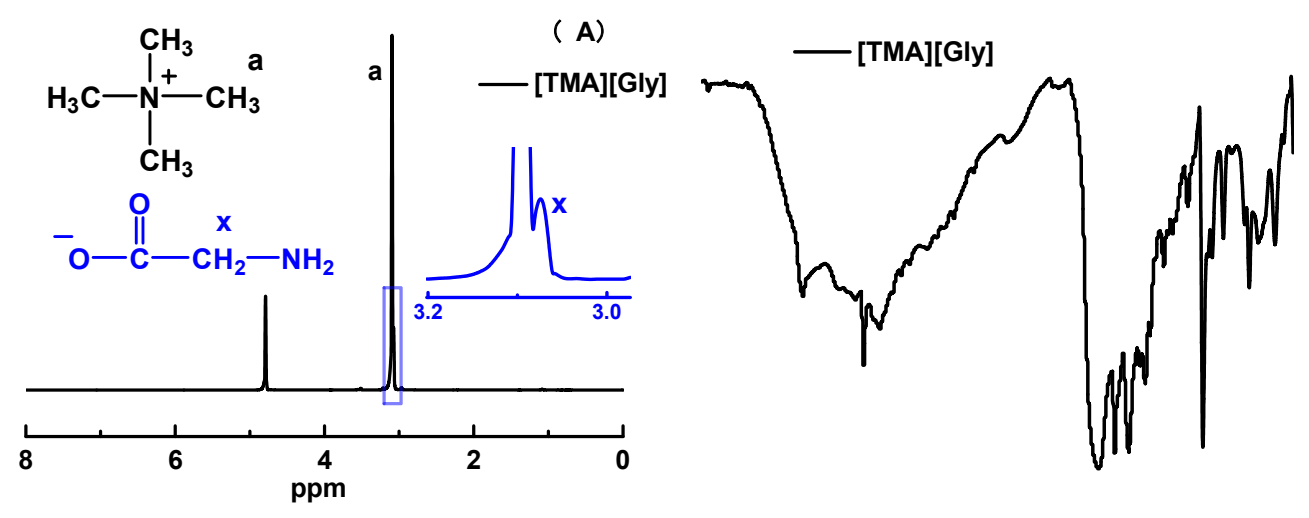

Figure 4. 1H-NMR spectra of TMA and [TMA][Gly] (A) and FT-IR of [TMA][Gly] (B). 
NMR and FT-IR were used to identify the chemical structures of AAILs synthesized in this work. As shown in Fig 4(A), proton signals of tetramethylammonium cation and glycine anion appeared at $\sim 3.095$ and 3.072 ppm (1 and 2), respectively, and the ratio of the area under signal 1 and 2 was 7:1, indicating that the neutralization rate was $85.7 \%$. Characteristic bands due to $\mathrm{C}-\mathrm{H}$ stretching at $\sim 3028$ and $2925 \mathrm{~cm}-1, \mathrm{C}-\mathrm{N}$ stretching at $\sim 952 \mathrm{~cm}-1$ of the cation and $\mathrm{C}=\mathrm{O}$ stretching at $1569 \mathrm{~cm}-1$ of the Glycine anion were seen in the spectra(Fig 4. B). The test results showed that the [TMA][Gly] had been synthesized successfully.
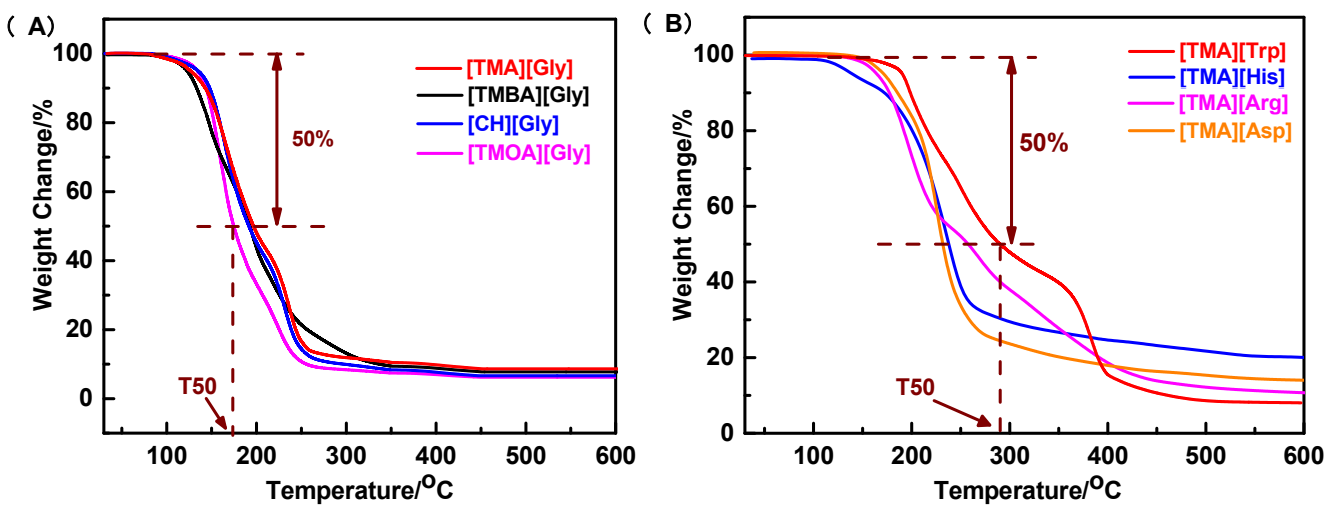

Figure 5. Thermal stability of AAILs. (A) thermal decomposition curve of Gly- based AAILs, T50 is the temperature, in which AAILs decomposed $50 \mathrm{wt} \%$ (B) thermal decomposition curve of TMAbased AAILs.

Table 1. T50 of AAILs.

\begin{tabular}{cc}
\hline AAILs & $\mathrm{T}_{50} /{ }^{\circ} \mathrm{C}$ \\
[TMOA][Gly] & 174.4 \\
[TMA][Gly] & 195.2 \\
[TMBA][Gly] & 191.5 \\
[CH][Gly] & 191.2 \\
[TMA][Asp] & 227.8 \\
[TMA][His] & 242.5 \\
[TMA][Arg] & 255.1 \\
[TMA][Trp] & 288.5 \\
\hline
\end{tabular}

Thermal stability of AAILs was analyzed by Thermogravimetric Analysis and the results were shown in Fig 5 and Table 1. From Table 1, we can see that different chemical structures led to different thermal stability. AAILs based on Gly- had the similar T 50 while the cations were different. And when the cation was TMA+, T50 differed due to different amino acid anions, and [TMA][Trp] achieved the highest thermal decomposition temperature.

\subsection{CO2 absorption experiment}

\subsubsection{CO2 absorption capacity of AAILs}

We investigated the $\mathrm{CO} 2$ sorption behaviors of various solvents made from [CH][Gly], [TMA][Gly], [TMOA][Gly] and [TMBA][Gly], respectively. The concentration of the four AAILs aqueous solutions were $2.5 \mathrm{~mol} / \mathrm{L}$. As illustrated in Figure 6(A), different AAILs aqueous solution had different $\mathrm{CO} 2$ absorption capacity. The solvent made from [TMBA][Gly] exhibited the highest $\mathrm{CO} 2$ sorption capacity reaching $0.83 \mathrm{~mol} \mathrm{CO} 2 / \mathrm{mol} \mathrm{IL}$, while the [TMA][Gly] had the lowest CO2 uptake of $0.4 \mathrm{~mol} \mathrm{CO} 2 / \mathrm{mol}$ IL. The absorption capacity of [CH][Gly] was $0.62 \mathrm{~mol} \mathrm{CO} 2 / \mathrm{mol}$ ILs, which was higher than [TMA][Gly] owing to the existence hydroxyl group(-OH) [6]. And the AAILs except [TMA][Gly] had higher CO2 sorption capacity than the theoretical value-0.5mol CO2/ILs according to the zwitterion mechanism[33]. The higher CO2 absorption ability of [TMOA][Gly] than of [TMA][Gly] shows that increasing the chains length can facilitate the CO2 capture. 

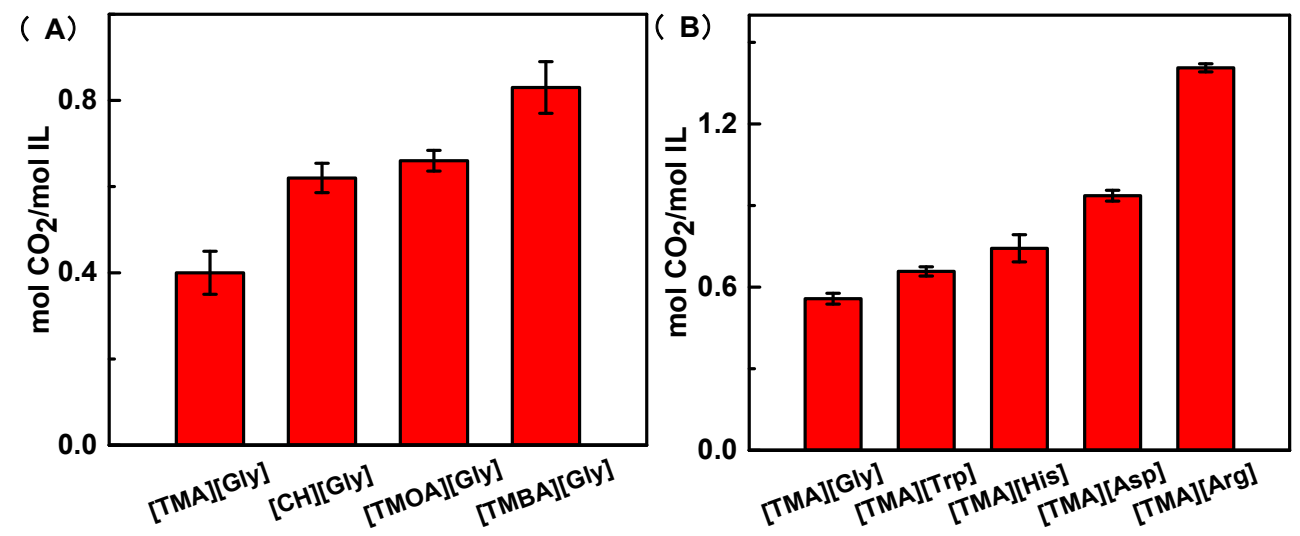

Figure 6. $\mathrm{CO} 2$ sorption of AAILs with different cations and anions. (A) $\mathrm{CO} 2$ absorption capacity of [CH][Gly], [TMA][Gly], [TMOA][Gly] and [TMBA][Gly] aqueous solutions of concentration of 2.5 $\mathrm{mol} / \mathrm{L}$ (B) $\mathrm{CO} 2$ absorption capacity of saturated aqueous solutions of [TMA][Gly], [TMA][Trp], [TMA][His], [TMA][Asp] and [TMA][Arg].

The saturated aqueous solutions of [TMA][Gly], [TMA][Trp], [TMA][His], [TMA][Asp] and [TMA][Asp] were also tested in this work. As shown in Figure 6(B), with the same cationtetramethylammonium, AAILs with different amino acid anions showed different $\mathrm{CO} 2$ absorption abilities. The saturated solution of [TMA][Arg] got the highest $\mathrm{CO} 2$ absorption amount per mole AAILs, because there were two primary and two secondary amine groups in one molecule[34]. There were two cations with one amino acid anion in [TMA][Asp], so that there may be "cross-linked" net, which contributed to the solubility of more $\mathrm{CO} 2$ molecule in the system. And the $\mathrm{CO} 2$ absorption capacity of [TMA][Asp] was $0.94 \mathrm{~mol} \mathrm{CO} / \mathrm{mol} \mathrm{ILs}$, which was the second highest among the saturated aqueous solutions of AAILs prepared in this work due to the interstitial spaces in [TMA][Asp] system.

\subsection{2. $\mathrm{CO} 2$ absorption with different concentrations of [TMA][Trp] aqueous solutions}
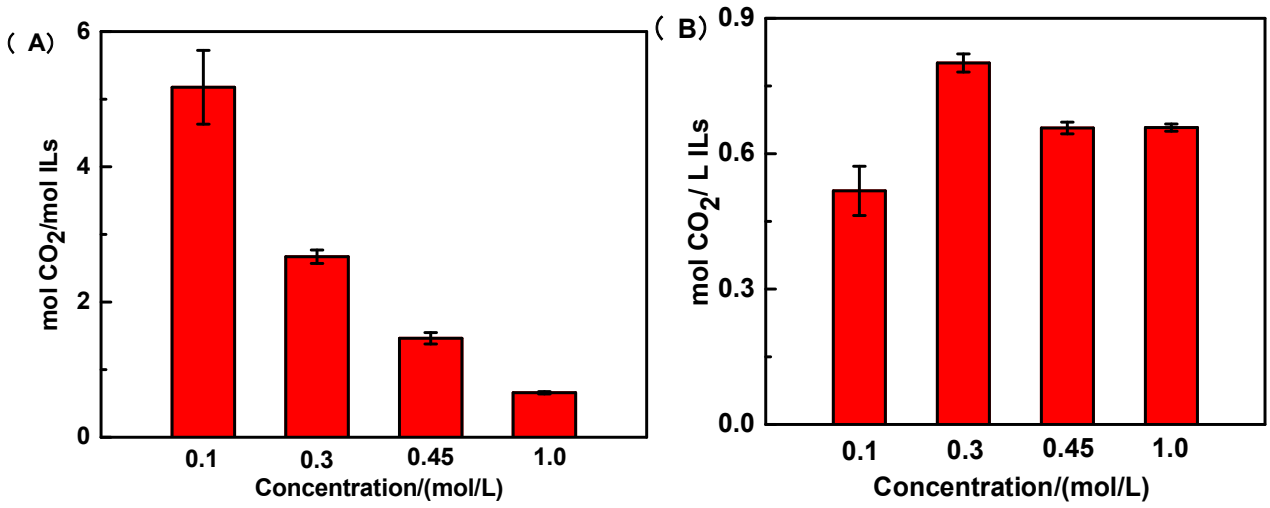

Figure 7. $\mathrm{CO} 2$ absorption capacity of [TMA][Trp] solutions of different concentrations. (A) $\mathrm{CO} 2$ absorption amount per mole AAILs; (B) CO2 absorption amount per unit volume.

[TMA][Trp] aqueous solutions of four concentrations $(0.1 \mathrm{M}, 0.3 \mathrm{M}, 0.45 \mathrm{M}$ and $1.0 \mathrm{M})$ were used for the $\mathrm{CO} 2$ bubbling experiment. As shown in Fig 7(A), all the solvents possessed excellent $\mathrm{CO} 2$ absorption. The $0.1 \mathrm{~mol} / \mathrm{L}$ [TMA][Trp] aqueous solution achieved the highest $\mathrm{CO} 2$ uptake amount of $5.176 \mathrm{~mol} \mathrm{CO} 2 / \mathrm{mol}$ AAILs without phase change, while the $0.45 \mathrm{~mol} / \mathrm{L}$ solvent had the lowest CO2 absorption capacity of $1.5 \mathrm{~mol} \mathrm{CO} / \mathrm{mol}$ AAILs with a significant phase change phenomenon. It can be seen in Fig 7(B), CO2 absorption capacity by per unit volume was increased along with the increase of concentration at lower concentration, and $\mathrm{CO} 2$ absorption capacity was not increased when the concentration continues to increase due to the quick formation of the solid phase. 


\subsection{Phase change in the $\mathrm{CO} 2$ bubbling experiment}

The liquid phase changed into solid-liquid phase in the process of bubbling CO2, as some articles have reported.

As illustrated in Fig 8 (A), all of the four AAILs of $2.5 \mathrm{~mol} / \mathrm{L}$ with different cations-[CH], [TMA], [TMOA] and [TMBA], of which the anion was Glycine, exhibited a phase-change phenomenon. Absorbent made from [TMOA][Gly] achieved the highest volume ratio between precipitation and solvents unloaded CO2, that was 0.213 , while the [TMA][Gly] got the smallest amount of solid phase. When the anion was Glycine, the volume of solid precipitated was related to the molecular structure of the cation, since the chemical and steric structure played a role on the solubility of the carbamate. Among the four cations used in this work, trimethyloctylammonium (TMOA) cation possessed a long hydrophobic alkyl chain. Thus, the carbamate loaded CO2 had the long hydrophobic parts at each end so that to yield more precipitation.
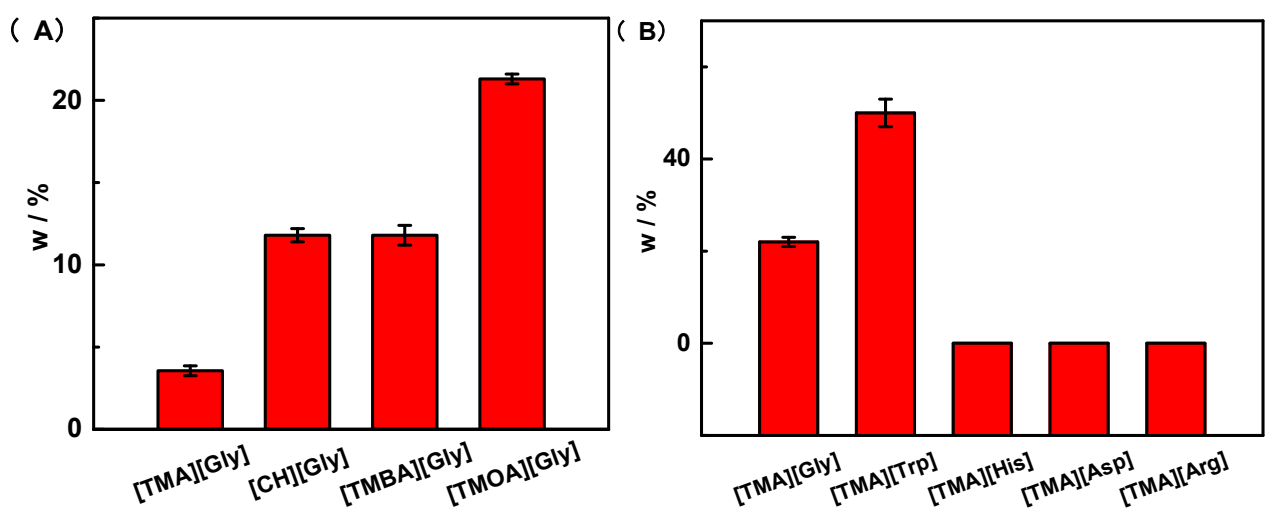

Figure 8. Phase change into solid-liquid in the $\mathrm{CO} 2$ uptake process. (A) volume ratio between precipitation and solvents before capturing $\mathrm{CO} 2$ in [CH][Gly], [TMA][Gly], [TMOA][Gly], [TMBA][Gly] aqueous solutions of $2.5 \mathrm{~mol} / \mathrm{L}$ (B) volume ratio between precipitation and solvents before capturing $\mathrm{CO} 2$ in saturated aqueous solutions of [TMA][Gly], [TMA][Trp], [TMA][His], [TMA][Asp] and [TMA][Arg].

As for the saturated solutions of the AAILs with different amino acid anions, there were no phase change in the system of [TMA][Asp] and [TMA][His] for the low concentration. Surprisingly, solvent of [TMA][Trp] exhibited a fast phase change and it was only 4 minutes to precipitated all the powder and it filled the whole solvent. So we carried out more experiments about [TMA][Trp]. The photo of phase change phenomenon of AAILs synthesized in this work was shown in Fig 9.

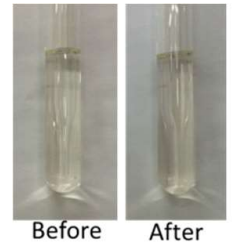

[TMA][Arg]

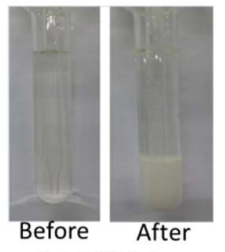

[TMA][Gly]

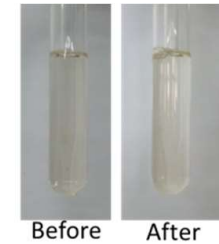

[TMA][Asp]

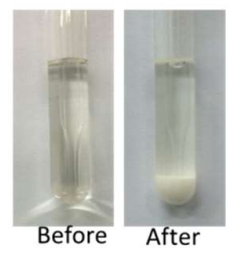

[CH][Gly]

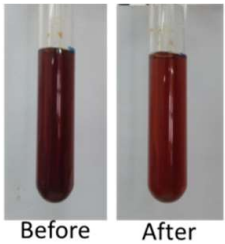

[TMA][His]

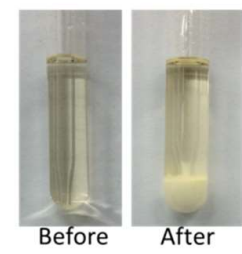

[TMBA][Gly]

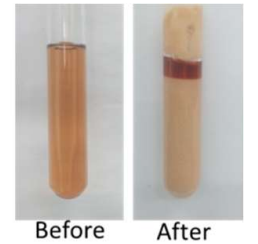

[TMA][Trp]

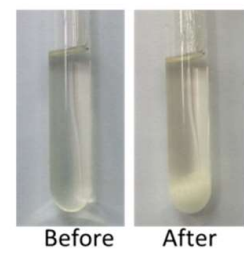

[TMOA][Gly]

Figure 9. Digtal photos of AAIL solvents of different concentrations before and after absorbing CO2. 


\subsection{Mechanism of phase change in [TMA][Gly] aqueous solution}<smiles>C[N+](C)(C)C</smiles><smiles>N[CH]C(=O)[O-]</smiles><smiles>CC(=O)NCC(=O)[O-]</smiles>

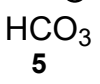

Figure 10. Chemical structures of the species in [TMA][Gly] absorbent system.

In order to get clear knowledge of the capture mechanism, 13C-NMR was used to analyze the species of the absorbing system, including [TMA][Gly], liquid phase, solid phase and the product stripped CO2. Chemical structures of the species were showed as Fig 10. The details of spectrums were presented in Fig 11.

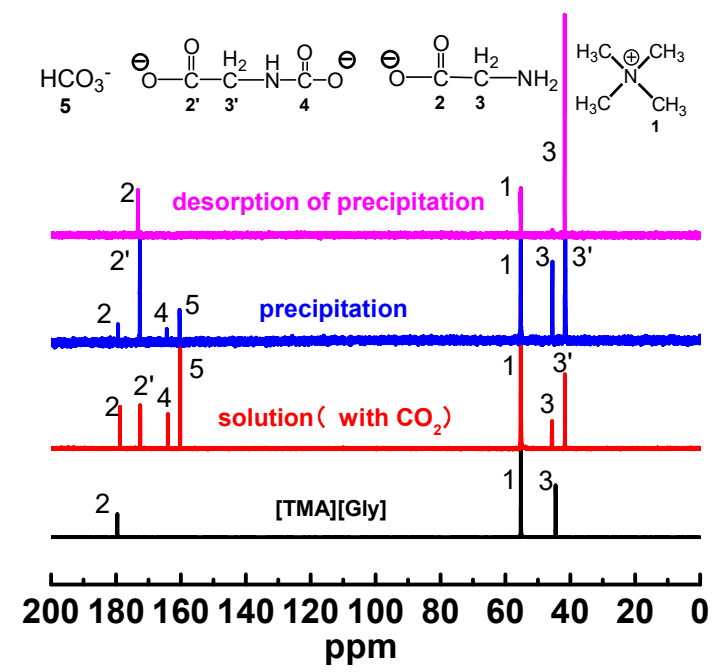

Figure 11. 13C-NMR spectra of [TMA][Gly] aqueous solution system before and after absorbing $\mathrm{CO} 2$.

It was obvious that new peaks appear in the 13C NMR spectrum of liquid phase and solid phase compared with the initial absorbent. The new signal (4) at $164 \mathrm{ppm}$ was corresponding to the carbamate carboxyl carbon species, and the peak (5) at 160 ppm was assigned to HCO3-[35-37]. Peaks associated with the Gly- shifted because of the existence of $\mathrm{H}+$. Both in the liquid phase and solid phase, the methylene carbon and carbonyl carbon of glycine shifted clearly. Compared with [TMA][Gly], there was no new peak in the spectrum of regeneration product of precipitation, which illustrated one successful desorption. Chemical shifts $(\delta C)$ of the each carbon atoms were summarized in Table 2.

Table 2. Chemical shift of 13C-NMR spectra $(\delta C)$ of $[\mathrm{TMA}][\mathrm{Gly}]$ absorbed system before and after capturing $\mathrm{CO} 2$ and the product of regeneration process.

\begin{tabular}{cccccccc}
\hline \multirow{2}{*}{ Species } & 1 & 2 & 2 & \multicolumn{7}{c}{$\delta \mathrm{C}(\mathrm{ppm})$} \\
[TMA][Gly] & 55.24 & 179.58 & $/$ & 44.54 & $/$ & $/$ & $/$ \\
solution & $55.25-55.20$ & 178.72 & 172.49 & 45.57 & 41.61 & 163.96 & 160.17 \\
precipitation & $55.26-55.20$ & 179.32 & 172.59 & 45.47 & 41.66 & 164.30 & 160.36 \\
desorption & $55.25-55.20$ & 173.20 & $/$ & 41.68 & $/$ & $/$ & $/$ \\
\hline
\end{tabular}

As showed in the 1H-NMR spectrums of Fig 12, carbamate appeared in both the liquid phase and solid phase, which was consistent with the result of 13C-NMR spectra. Due to the pH change of the solution after capturing $\mathrm{CO} 2$, the signals of $\mathrm{H}$ atoms in -OOC-CH2-NH2/-OOC-CH2-NH3+ shifted[31]. 


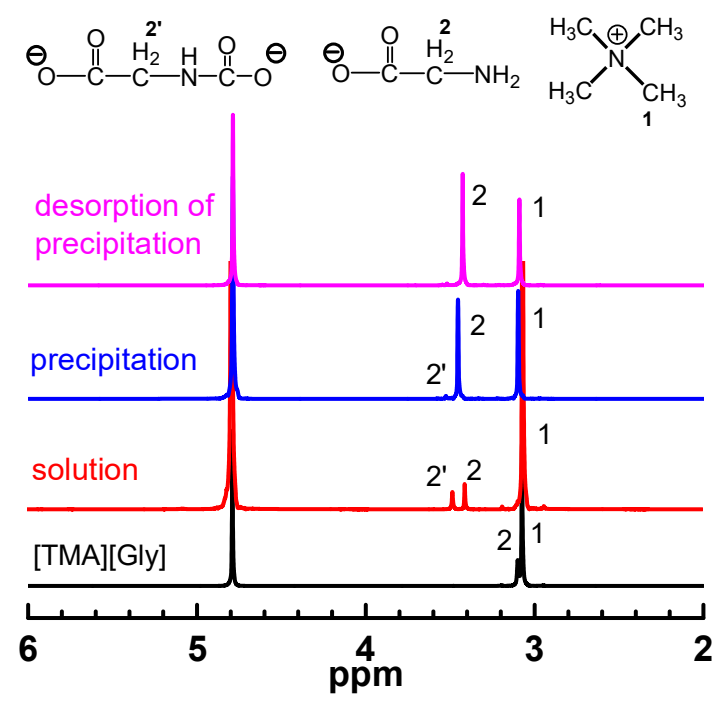

Figure 12. 1H-NMR spectra of [TMA][Gly] aqueous solution system before and after capturing CO2.

In order to get the proportion of each species, liquid phase after complete phase separation had been heated to release $\mathrm{CO}$, rotary evaporated and vacuum drying, successively. The proportion of [TMA][Gly] in the liquid was $62 \mathrm{wt} \%$ of total addition. It easy to get that the proportion of [TMA][Gly] in the solid phase was $38 \mathrm{wt} \%$, while the volume ratio of solid was $22 \%$. According to the integral area of peaks in 1H-NMR spectrum, the ratio of different species could be obtained. It had been calculated that the weight proportion of -OOC-CH2-NH2, -OOC-CH2-NH-COO- and $(\mathrm{CH} 3) 3 \mathrm{~N}+$ in liquid phase and solid phase, respectively. It can be seen in Fig 2.8 that most of the carbamate was in the liquid phase, only a small part precipitated. Due to the intermolecular force, some of the initial absorbent- [TMA][Gly] was precipitated.

Table 3. Ratio of different species in liquid phase and solid phase to the total weight of each species.

\begin{tabular}{cccc}
\hline \multirow{2}{*}{ Species } & \multicolumn{3}{c}{ Ratio/wt\% } \\
& $-\mathrm{OOC}-\mathrm{CH}_{2}-\mathrm{NH}_{2} /-\mathrm{OOC}-\mathrm{CH}_{2}-\mathrm{NH}_{3}{ }^{+}$ & $-\mathrm{OOC}-\mathrm{CH}_{2}-\mathrm{NH}-\mathrm{COO}{ }^{-}$ & $\left(\mathrm{CH}_{3}\right)_{3} \mathrm{~N}^{+}$ \\
solution & 25.17 & 79.68 & 82.47 \\
precipitation & 74.83 & 20.32 & 17.53 \\
\hline
\end{tabular}

According to the analysis of NMR spectra, it could be regarded that mechanism of [TMA][Gly] aqueous solution capturing $\mathrm{CO} 2$ was similar with that of amine absorbents in the first stage[38-39]. When CO2 was captured into [TMA][Gly] aqueous solution, two functional anions of Gly- (2 -OOC$\mathrm{CH} 2-\mathrm{NH} 2)$ was reacted with one $\mathrm{CO} 2$ molecule firstly forming one carbamate (-OOC-CH2-NHCOO-) group and one corresponding cationic group (-OOC-CH2-NH3+). Due to the existence of water, in the second period, carbamate was easily hydrolyzed to HCO3- and Gly-[40]. The reactions balanced each other. We can infer that if all the carbamate hydrolyzed, the absorption capacity by per mole [TMA][Gly] would follow 1:1 stoichiometry. That indicated absorption capacity of CO2 was 0.5-1 mol CO2 / mol AAILs theoretically in the [TMA][Gly] aqueous solution. As the CO2 capture into absorbent proceeded, the amount of carbamate and carbonate species in solution increased. Due to the low solubility of the two species in water, carbamate and carbonate species easily precipitated from the liquid phase. At the same time, intermolecular force and decrease of the $\mathrm{pH}$ led to a portion of glycine and [TMA][Gly] precipitated. In the regeneration process, $\mathrm{HCO}$ - reacted with -OOC-CH2$\mathrm{NH} 3+$ to release $\mathrm{CO} 2$ when heated. Proposed reaction mechanism in AAILs aqueous solution was illustrated as follows: 


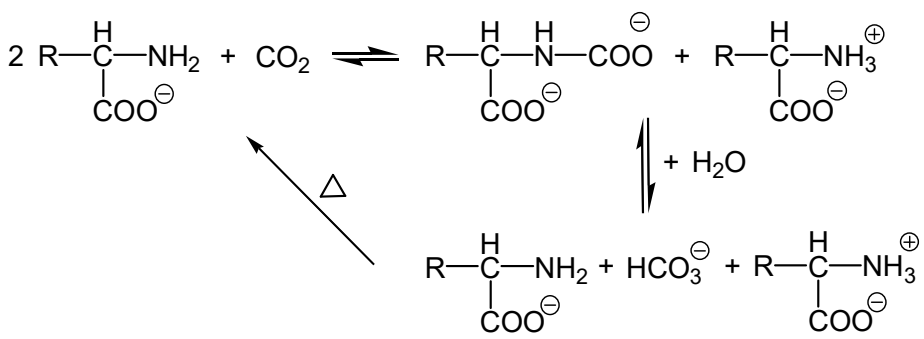

\subsection{Regeneration performance [TMA][Trp] solution}

The [TMA][Trp] absorbent of $0.45 \mathrm{~mol} / \mathrm{L}$ was chosen to investigate the regeneration performance in the cycle of absorption and desorption because of its moderate absorption capacity and phase change performance. Here, different two desorption methods were adopted as was stated above-one is by heating the whole absorbent captured $\mathrm{CO} 2$ in $100 \mathrm{oC}$ atomosohere for 6 hours, and another is by only heating the solid phase in $120 \mathrm{oC}$ for 6 hours.

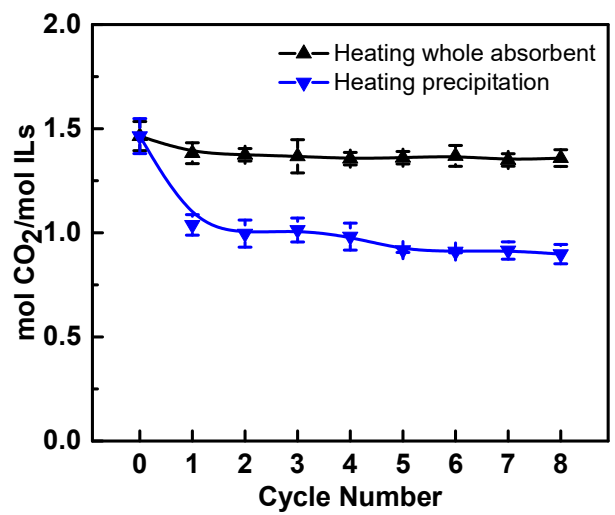

Figure 13. The absorption capacity of [TMA][Trp] in the absorption and desorption cycle by different regeneration methods.

The results were showed in Fig 10. The regeneration rate of stripping by heating the whole solvent was $93 \%$. When the desorption experiment was done by only heating the solid phase, the $\mathrm{CO} 2$ absorption capacity was decreased to $1.04 \mathrm{~mol} \mathrm{CO} / \mathrm{mol}$ AAILs in the first cycle from $1.46 \mathrm{~mol}$ $\mathrm{CO} 2 / \mathrm{mol}$ AAILs, which indicated that the regeneration rate was $71 \%$. And then, the regeneration rate was decreased to and retained to $62 \%$. There were small part of carbamate / HCO3- existed in the liquid phase. However, the absorbent still presented good performance of regeneration and reuse with low regeneration energy consumption.

\section{Conclusions}

A serial of amino acid ionic liquids were synthesized and characterized. Their $\mathrm{CO} 2$ absorption and phase change behaviors were investigated. All the prepared AAILs aqueous solutions exhibit significant $\mathrm{CO} 2$ absorption capacity. Then [TMA][Trp] was selected as the most favorable solvent for its excellent phase change behavior after capturing $\mathrm{CO} 2$. By heating only the solid $\mathrm{CO} 2$-rich phase to desorption temperature, the [TMA][Trp] absorbent can recycle and reuse many times. Regenerating the solid phase while reusing the liquid phase without any desorption can reduce regeneration energy and save resources.

Acknowledgment: We acknowledge financial support from National Natural Science Foundation of China (51503060) and Hubei Nature Science Foundation of China (2014CFB549).

Conflict of interest: The authors declare that they have no conflict of interest. 


\section{References}

[1] Knight J, Harrison S. The Impacts of Climate Change on Terrestrial Earth Surface Systems [J]. Nature Climate Change, 2013, 3(1):24-29.

[2] Zhang F, Fang C G, Wu Y T, et al. Absorption of CO2 in the aqueous solutions of functionalized ionic liquids and MDEA[J]. Chemical Engineering Journal, 2010, 160(2):691-697.

[3] Xue Z, Zhang Z, Han J, et al. Carbon dioxide capture by a dual amino ionic liquid with amino-functionalized imidazolium cation and taurine anion[J]. International Journal of Greenhouse Gas Control, 2011, 5(4):628633.

[4] Song H J, Park S, Kim H, et al. Carbon dioxide absorption characteristics of aqueous amino acid salt solutions[J]. International Journal of Greenhouse Gas Control, 2012, 11(6):64-72.

[5] Aronu U E, Hessen E T, Haug-Warberg T, et al. Equilibrium absorption of carbon dioxide by amino acid salt and amine amino acid salt solutions[J]. Energy Procedia, 2011, 4(1):109-116.

[6] Lv B, Xia Y, Shi Y, et al. A novel hydrophilic amino acid ionic liquid [C2OHmim][Gly] as aqueous sorbent for CO2 capture[J]. International Journal of Greenhouse Gas Control, 2016, 46:1-6.

[7] Carvalho P J, Coutinho J A P. On the Nonideality of CO2 Solutions in Ionic Liquids and Other Low Volatile Solvents[M]. 2010:774-780.

[8] Perezblanco M E, Maginn E J. Molecular Dynamics Simulations of CO2 at an Ionic Liquid Interface: Adsorption, Ordering, and Interfacial Crossing[J]. Journal of Physical Chemistry B, 2010, 114(36):11827-37.

[9] Shiflett M B, Niehaus A M S, Yokozeki A. Separation of CO2 and H2S Using Room-Temperature Ionic Liquid [bmim][MeSO4][J]. J.chem.eng.data, 2010, 55(11):4785-4793.

[10] Lin H, Bai P, Guo X. Ionic Liquids for SO2 Capture: Development and Progress[J]. Asian Journal of Chemistry, 2014, 26(9):2501-2506.

[11] Zhao Y, Zhang X, Zhen Y, et al. Novel alcamines ionic liquids based solvents: Preparation, characterization and applications in carbon dioxide capture[J]. International Journal of Greenhouse Gas Control, 2011, 5(2):367-373.

[12] Ramdin M, Loos T W D, Vlugt T J H. State-of-the-Art of CO2 Capture with Ionic Liquids[J]. Industrial \& Engineering Chemistry Research, 2012, 51(24):8149-8177.

[13] Mi M W, Hao Y Z, Yan Y L, et al. Novel CO2-Capture Derived from the Basic Ionic Liquids Orientated on Mesoporous Materials[J]. Acs Applied Materials \& Interfaces, 2014,6(15):12947-12955.

[14] Zulfiqar S, Sarwar M I, Mecerreyes D. Polymeric ionic liquids for CO2 capture and separation: potential, progress and challenges[J]. Polym Chem, 2015, 6(36):6435-6451.

[15] Hu Y F, Liu Z C, Xu C M, et al. The Molecular Characteristics Dominating the Solubility of Gases in Ionic Liquids[J]. Chemical Society Reviews, 2011, 40(7):3802-23.

[16] Zulfiqar S, Sarwar M I, Mecerreyes D. Polymeric ionic liquids for CO2 capture and separation: potential, progress and challenges[J]. Polym Chem, 2015, 6(36):6435-6451.

[17] Carlisle T K, Bara J E, Gabriel C J, et al. Interpretation of CO2 Solubility and Selectivity in NitrileFunctionalized Room-Temperature Ionic Liquids Using a Group Contribution Approach[J]. Industrial \& Engineering Chemistry Research, 2008, 47(18):7005-7012.

[18] Gardas R L, Ge R, Goodrich P, et al. Thermophysical Properties of Amino Acid-Based Ionic Liquids[J]. Journal of Chemical \& Engineering Data, 2009, 55(4):1505-1515.

[19] Zhang X, Zhang X, Dong H, et al. Carbon capture with ionic liquids: overview and progress[J]. Energy \& Environmental Science, 2012, 5(5):6668-6681.

[20] Dai Z, Noble R D, Gin D L, et al. Combination of ionic liquids with membrane technology: A new approach for CO2 separation[J]. Journal of Membrane Science, 2016, 497:1-20.

[21] Hiremath $V$, Jadhav A $H$, Lee $H$, et al. Highly reversible CO2 capture using amino acid functionalized ionic liquids immobilized on mesoporous silica[J]. Chemical Engineering Journal, 2015, 287:602-617.

[22] Zhang F, Ma J W, Zhou Z, et al. Study on the absorption of carbon dioxide in high concentrated MDEA and ILs solutions[J]. Chemical Engineering Journal, 2011, 181-182(2):222-228.

[23] Gao Y, Zhang F, Huang K, et al. Absorption of CO2 in amino acid ionic liquid (AAIL) activated MDEA solutions[J]. International Journal of Greenhouse Gas Control, 2013, 19(21):379-386.

[24] Yu H, Imamura K, Yue M, et al. Reversible Absorption of CO2 Triggered by Phase Transition of AmineContaining Micro- and Nanogel Particles[J]. Journal of the American Chemical Society, 2012, 134(44):1817780. 
[25]Hasib-Ur-Rahman M, Siaj M, Larachi F. CO2 capture in alkanolamine/room-temperature ionic liquid emulsions: A viable approach with carbamate crystallization and curbed corrosion behavior [J]. International Journal of Greenhouse Gas Control, 2012, 6(3):246-252.

[26] Hasiburrahman M, Larachi F. CO2 Capture in Alkanolamine-RTIL Blends via Carbamate Crystallization: Route to Efficient Regeneration[J]. Environmental Science \& Technology, 2012, 46(20):11443-50.

[27] Pinto D D D, Zaidy S A H, Hartono A, et al. Evaluation of a phase change solvent for CO2 capture: Absorption and desorption tests[J]. International Journal of Greenhouse Gas Control, 2014, 28(9):318-327.

[28] Hasiburrahman M, Larachi F. CO2 Capture in Alkanolamine-RTIL Blends via Carbamate Crystallization: Route to Efficient Regeneration[J]. Environmental Science \& Technology, 2012, 46(20):11443-50.

[29] Feron P H M. New solvents based on amino-acid salts for $\mathrm{CO} 2$ capture from flue gases[J]. Greenhouse Gas Control Technologies 7, 2005,2(1):1153-58.

[30] Zheng S, Tao M, Liu Q, et al. Capturing CO2 into the precipitate of a phase-changing solvent after absorption.[J]. Environmental Science \& Technology, 2014, 48(15):8905-10.

[31] Wang X, Akhmedov N G, Hopkinson D, et al. Phase change amino acid salt separates into CO2-rich and CO2-lean phases upon interacting with CO2[J]. Applied Energy, 2016, 161:41-47.

[32] Jiang Y Y, Wang G N, Zhou Z, et al. Tetraalkylammonium amino acids as functionalized ionic liquids of low viscosity.[J]. Chemical Communications, 2008, 8(4):505-507.

[33] Jiang B, Wang X, Gray M M L, et al. Development of amino acid and amino acid-complex based solid sorbents for CO2, capture[J]. Applied Energy, 2013, 109 (2):112-118.

[34] Sistla Y S, Khanna A. CO2 absorption studies in amino acid-anion based ionic liquids[J]. Chemical Engineering Journal, 2015, 273:268-276

[35] Lv B H, Jing G H, Qian Y H, et al. An efficient absorbent of amine-based amino acid-functionalized ionic liquids for CO2 capture: High capacity and regeneration ability[J]. Chemical Engineering Journal, 2016, 289:212-218.

[36] Hartono A, Aronu U E, Svendsen H F. Liquid speciation study in amine amino acid salts for CO2 absorbent with 13C-NMR[J]. Energy Procedia, 2011, 4: 209-215.

[37] Guo B S, Jing G H, Zhou Z M. Regeneration performance and absorption/desorption mechanism of tetramethylammonium glycinate aqueous solution for carbon dioxide capture[J]. International Journal of Greenhouse Gas Control, 2015, 34: 31-38.

[38] Zhang F, Gao Y, Wu X K, et al. Regeneration performance of amino acid ionic liquid (AAIL) activated MDEA solutions for CO2 capture[J]. Chemical Engineering Journal, 2013,223:371-378.

[39] Zhang Y, Wu Z K, Chen S L, et al. CO2 capture by imidazolate-based ionic liquids: effect of functionalized cation and dication[J]. Industrial \& Engineering Chemistry Research, 2013, 52:6069-6075.

[40] Caplow M. Kinetics of Carbamate Formation and Breakdown[J] Journal of the American Chemical Society, 1968, 90: 6795-6803. 